\title{
Binary linear complementary dual codes
}

\author{
Masaaki Harada*and Ken Saito ${ }^{\dagger}$
}

Dedicated to Professor Masahiko Miyamoto on His 65th Birthday

\begin{abstract}
Linear complementary dual codes (or codes with complementary duals) are codes whose intersections with their dual codes are trivial. We study binary linear complementary dual $[n, k]$ codes with the largest minimum weight among all binary linear complementary dual $[n, k]$ codes. We characterize binary linear complementary dual codes with the largest minimum weight for small dimensions. A complete classification of binary linear complementary dual $[n, k]$ codes with the largest minimum weight is also given for $1 \leq k \leq n \leq 16$.
\end{abstract}

\section{Introduction}

An $[n, k]$ code $C$ over $\mathbb{F}_{q}$ is a $k$-dimensional vector subspace of $\mathbb{F}_{q}^{n}$, where $\mathbb{F}_{q}$ denotes the finite field of order $q$ and $q$ is a prime power. A code over $\mathbb{F}_{2}$ is called binary. The parameters $n$ and $k$ are called the length and dimension of $C$, respectively. The weight wt $(x)$ of a vector $x \in \mathbb{F}_{q}^{n}$ is the number of nonzero components of $x$. A vector of $C$ is called a codeword of $C$. The minimum non-zero weight of all codewords in $C$ is called the minimum weight $d(C)$ of $C$ and an $[n, k]$ code with minimum weight $d$ is called an $[n, k, d]$ code. Two $[n, k]$ codes $C$ and $C^{\prime}$ over $\mathbb{F}_{q}$ are equivalent, denoted $C \cong C^{\prime}$, if there is an $n \times n$ monomial matrix $P$ over $\mathbb{F}_{q}$ with $C^{\prime}=C \cdot P=\{x P \mid x \in C\}$.

\footnotetext{
*Research Center for Pure and Applied Mathematics, Graduate School of Information Sciences, Tohoku University, Sendai 980-8579, Japan. email: mharada@m.tohoku.ac.jp.

${ }^{\dagger}$ Research Center for Pure and Applied Mathematics, Graduate School of Information Sciences, Tohoku University, Sendai 980-8579, Japan. email: kensaito@ims.is.tohoku.ac.jp.
} 
The dual code $C^{\perp}$ of a code $C$ of length $n$ is defined as $C^{\perp}=\left\{x \in \mathbb{F}_{q}^{n} \mid\right.$ $x \cdot y=0$ for all $y \in C\}$, where $x \cdot y$ is the standard inner product. A code $C$ is called linear complementary dual (or a linear code with complementary dual) if $C \cap C^{\perp}=\left\{\mathbf{0}_{n}\right\}$, where $\mathbf{0}_{n}$ denotes the zero vector of length $n$. We say that such a code is LCD for short.

LCD codes were introduced by Massey [11] and gave an optimum linear coding solution for the two user binary adder channel. LCD codes are an important class of codes for both theoretical and practical reasons (see [2], [3], 4], 6], [7], 9], [10], [11, [12], [13]). It is a fundamental problem to classify LCD $[n, k]$ codes and determine the largest minimum weight among all LCD $[n, k]$ codes. Recently, much work has been done concerning this fundamental problem (see [3], 4], 6], [7], [10]). In particular, we emphasize the recent work by Carlet, Mesnager, Tang, Qi and Pellikaan [4]. It has been shown in [4] that any code over $\mathbb{F}_{q}$ is equivalent to some LCD code for $q \geq 4$. This motivates us to study binary LCD codes.

Throughout this paper, let $d(n, k)$ denote the largest minimum weight among all binary LCD $[n, k]$ codes. Recently, some bounds on the minimum weights of binary LCD $[n, k]$ codes have been established in [7]. More precisely, $d(n, 2)$ has been determined and the values $d(n, k)$ have been calculated for $1 \leq k \leq n \leq 12$. In this paper, we characterize binary LCD $[n, k, d(n, k)]$ codes for small $k$. The concept of $k$-covers of $m$-sets plays an important role in the study of such codes. Using the characterization, we give a classification of binary $\operatorname{LCD}[n, 2, d(n, 2)]$ codes and we determine $d(n, 3)$. In this paper, a complete classification of binary LCD $[n, k]$ codes having the minimum weight $d(n, k)$ is also given for $1 \leq k \leq n \leq 16$.

The paper is organized as follows. In Section 2, definitions, notations and basic results are given. We also give a classification of binary LCD $[n, k, d(n, k)]$ codes for $k=1, n-1$. In Section 3, we give some characterization of binary LCD codes using $k$-covers of $m$-sets. This characterization is used in Sections 4 and 5. In Section 4, we study binary LCD codes of dimension 2. We give a classification of binary $\operatorname{LCD}[n, 2, d(n, 2)]$ codes for $n=6 t(t \geq 1), 6 t+1(t \geq 1), 6 t+2(t \geq 0), 6 t+3(t \geq 1), 6 t+4(t \geq 0)$ and $6 t+5(t \geq 0)$ (Theorems 4.5 and 4.8). In Section 5, we study binary LCD codes of dimension 3. In Section 5 , we show that $d(n, 3)=\left\lfloor\frac{4 n}{7}\right\rfloor$ if $n \equiv 3,5$ $(\bmod 7)$ and $\left\lfloor\frac{4 n}{7}\right\rfloor-1$ otherwise, for $n \geq 3$ (Theorem 5.1). We also establish the uniqueness of binary $\operatorname{LCD}[n, 3, d(n, 3)]$ codes for $n \equiv 0,2,3,5(\bmod 7)$. Finally, in Section 6, we give a complete classification of binary LCD $[n, k]$ codes having the minimum weight $d(n, k)$ for $2 \leq k \leq n-1 \leq 15$. 
All computer calculations in this paper were done with the help of MAGma [1].

\section{Preliminaries}

\subsection{Definitions, notations and basic results}

Throughout this paper, $\mathbf{0}_{s}$ and $\mathbf{1}_{s}$ denote the zero vector and the all-one vector of length $s$, respectively. Let $I_{k}$ denote the identity matrix of order $k$ and let $A^{T}$ denote the transpose of a matrix $A$.

From now on, all codes mean binary. Let $C$ be an $[n, k]$ code. The weight enumerator of $C$ is given by $\sum_{i=0}^{n} A_{i} y^{i}$, where $A_{i}$ is the number of codewords of weight $i$ in $C$. It is trivial that two codes with distinct weight enumerators are inequivalent. The dual code $C^{\perp}$ of $C$ is defined as $C^{\perp}=\left\{x \in \mathbb{F}_{2}^{n} \mid\right.$ $x \cdot y=0$ for all $y \in C\}$, where $x \cdot y$ is the standard inner product. A code $C$ is called linear complementary dual (or a linear code with complementary dual) if $C \cap C^{\perp}=\left\{\mathbf{0}_{n}\right\}$. We say that such a code is LCD for short. A generator matrix of $C$ is a $k \times n$ matrix whose rows are basis vectors of $C$. A parity-check matrix of $C$ is a generator matrix of $C^{\perp}$. The following characterization is due to Massey [11].

Proposition 2.1. Let $C$ be a code. Let $G$ and $H$ be a generator matrix and a parity-check matrix of $C$, respectively. Then the following properties are equivalent:

(i) $C$ is $L C D$,

(ii) $C^{\perp}$ is $L C D$,

(iii) $G G^{T}$ is nonsingular,

(iv) $H H^{T}$ is nonsingular.

Throughout this paper, the condition (iii) is used to verify that a given code is LCD.

Let $d(n, k)$ denote the largest minimum weight among all LCD $[n, k]$ codes throughout this paper.

Lemma 2.2. Suppose that there is an $L C D[n, k, d]$ code $C$. If $d(n-1, k) \leq$ $d-1$, then $d\left(C^{\perp}\right) \geq 2$. 
Proof. Suppose that $d\left(C^{\perp}\right)=1$. Then some column of a generator matrix of $C$ is $\mathbf{0}_{k}$. By deleting the column, an LCD $[n-1, k, d]$ code is constructed.

Lemma 2.3. Suppose that there is an $L C D[n, k, d]$ code $C$ with $d\left(C^{\perp}\right) \geq 2$. If $n-k \geq 2^{k}$, then there is an $L C D[n-2, k]$ code $D$ with $d\left(D^{\perp}\right) \geq 2$.

Proof. We may assume without loss of generality that $C$ has generator matrix of the form $G=\left(\begin{array}{cc}I_{k} & M\end{array}\right)$, where $M$ is a $k \times(n-k)$ matrix. Since $d\left(C^{\perp}\right) \geq$ 2, no column of $M$ is $\mathbf{0}_{k}$. Since $n-k \geq 2^{k}$, some two columns of $M$ are identical. Let $G^{\prime}$ be the matrix obtained from $G$ by deleting the two columns. By Proposition 2.1 (iii), the code with generator matrix $G^{\prime}$ is LCD.

The above lemmas are used in Sections 3, 4 and 5.

\subsection{LCD codes of dimensions $1, n-1$}

It is trivial that $\mathbb{F}_{2}^{n}$ is an $\operatorname{LCD}[n, n, 1]$ code. It is known [6] that

$$
(d(n, 1), d(n, n-1))= \begin{cases}(n, 2) & \text { if } n \text { is odd } \\ (n-1,1) & \text { if } n \text { is even }\end{cases}
$$

The following propositions are trivial, so we omit the straightforward proofs.

Proposition 2.4. There is a unique $L C D[n, 1, d(n, 1)]$ code, up to equivalence.

Proposition 2.5. (i) Suppose that $n$ is odd. Then there is a unique LCD $[n, n-1,2]$ code, up to equivalence.

(ii) Suppose that $n$ is even. Then there are $n / 2$ inequivalent $L C D[n, n-1,1]$ codes.

\section{Constructions of LCD codes from $k$-covers}

In this section, we study LCD codes constructed from $k$-covers of $m$-sets. We give a characterization of LCD codes of dimensions 2 and 3 using $k$-covers. 


\subsection{LCD codes from $k$-covers}

Let $m$ and $k$ be positive integers. Let $X$ be a set with $m$ elements (for short $m$-set). A $k$-cover of $X$ is a collection of $k$ not necessarily distinct subsets of $X$ whose union is $X[5]$. This concept plays an important role in the study of LCD codes for small dimensions.

We define a generator matrix from a $k$-cover $\left\{Y_{1}, Y_{2}, \ldots, Y_{k}\right\}$ of an $m$ set $X=\{1,2, \ldots, m\}$ as follow. Since the matrix depends on the ordering chosen for $Y_{1}, Y_{2}, \ldots, Y_{k}$, in this paper, we fix the order. More precisely, we define a $k$-cover as a sequence $\mathcal{Y}=\left(Y_{1}, Y_{2}, \ldots, Y_{k}\right)$. Let $\mathcal{Y}=\left(Y_{1}, Y_{2}, \ldots, Y_{k}\right)$ be a $k$-cover of $X$. We define the following subsets of $\{1,2, \ldots, k+\ell m\}$ :

$$
\begin{aligned}
Z_{1} & =\{1\} \cup\left(k+Y_{1}\right) \cup\left(k+m+Y_{1}\right) \cup \cdots \cup\left(k+(\ell-1) m+Y_{1}\right), \\
Z_{2} & =\{2\} \cup\left(k+Y_{2}\right) \cup\left(k+m+Y_{2}\right) \cup \cdots \cup\left(k+(\ell-1) m+Y_{2}\right), \\
& \vdots \\
Z_{k} & =\{k\} \cup\left(k+Y_{k}\right) \cup\left(k+m+Y_{k}\right) \cup \cdots \cup\left(k+(\ell-1) m+Y_{k}\right),
\end{aligned}
$$

where $\ell$ is an even positive integer and $a+Y_{i}=\left\{a+y \mid y \in Y_{i}\right\}$ for a positive integer $a$. Let $S$ be a subset of $\{1,2, \ldots, s\}$. We define the binary vector $x=\left(x_{1}, x_{2}, \ldots, x_{s}\right)$, where $x_{i}=1$ if $i \in S$ and $x_{i}=0$ otherwise. This vector $x$ is called the characteristic vector of $S$. Let $z_{i}$ be the characteristic vector of $Z_{i}(i=1,2, \ldots, k)$. Then define the $k \times(k+\ell m)$ matrix $G(\mathcal{Y})$ such that $z_{i}$ is the $i$-th row. We denote the code with generator matrix of the form $G(\mathcal{Y})$ by $C(\mathcal{Y})$.

Proposition 3.1. The code $C(\mathcal{Y})$ is an $L C D[\ell m+k, k]$ code with $d\left(C(\mathcal{Y})^{\perp}\right)=$ 2.

Proof. Since $\ell$ is even, $G(\mathcal{Y}) G(\mathcal{Y})^{T}=I_{k}$. Thus, $C(\mathcal{Y})$ is LCD. Since $\mathcal{Y}$ is a $k$-cover of $X$, no column of $G(\mathcal{Y})$ is $\mathbf{0}_{k}$ and some two columns of $G(\mathcal{Y})$ are identical. This implies that $d\left(C(\mathcal{Y})^{\perp}\right)=2$.

Now we consider the case $k=2,3$ and $\ell=2$. Let $\mathcal{Y}$ be a 2 -cover and a 3 cover of $X$, respectively. Let $C(\mathcal{Y})$ be a $[2 m+2,2]$ code and a $[2 m+3,3]$ code with generator matrices of the form $G(\mathcal{Y})$, respectively. Let $C^{\prime}(\mathcal{Y})$ denote the $[2 m+3,2]$ code and the $[2 m+4,3]$ code with generator matrices of the following form:

$$
G^{\prime}(\mathcal{Y})=\left(\begin{array}{ll}
G(\mathcal{Y}) & 1 \\
& 1
\end{array}\right) \text { and }\left(\begin{array}{ll}
G(\mathcal{Y}) & 1 \\
& 1
\end{array}\right)
$$


respectively.

Proposition 3.2. The code $C^{\prime}(\mathcal{Y})$ is $L C D$.

Proof. For $k=2$ and 3, the result follows from

$$
G^{\prime}(\mathcal{Y}) G^{\prime}(\mathcal{Y})^{T}=\left(\begin{array}{cc}
0 & 1 \\
1 & 0
\end{array}\right) \text { and }\left(\begin{array}{ccc}
1 & 0 & 0 \\
0 & 0 & 1 \\
0 & 1 & 0
\end{array}\right)
$$

respectively.

The above proposition is used in Propositions 3.4 and 3.6 .

\subsection{LCD codes from 2-covers}

In this subsection, we show that $\operatorname{LCD}[n, 2]$ codes $C$ with $d\left(C^{\perp}\right) \geq 2$ are constructed from 2-covers for $n=2 m+2,2 m+3(m \geq 1)$.

Proposition 3.3. Suppose that $m \geq 1$. Let $C$ be an $L C D[2 m+2,2]$ code with $d\left(C^{\perp}\right) \geq 2$. Then there is a 2-cover $\left(Y_{1}, Y_{2}\right)$ of an $m$-set $X$ such that $C \cong C\left(\left(Y_{1}, Y_{2}\right)\right)$.

Proof. We may assume without loss of generality that $C$ has generator matrix of the following form:

$$
\left(\begin{array}{lll}
1 & 0 & \\
0 & 1 & M
\end{array}\right)
$$

where $M$ is a $2 \times 2 m$ matrix such that no column is $\mathbf{0}_{2}$. If $2 m \geq 4$, then some two columns of $M$ are identical. Hence, an LCD [2m,2] code is constructed by Lemma 2.3. By continuing this process, an LCD $[4,2]$ code with generator matrix of the form (1) is constructed. Hence, we show that such a code is constructed from a 2-cover.

Since no column of $M$ is $\mathbf{0}_{2}$, it is sufficient to consider the [4,2] codes with generator matrices (1), where

$$
M=\left(\begin{array}{ll}
0 & 0 \\
1 & 1
\end{array}\right),\left(\begin{array}{ll}
0 & 1 \\
1 & 0
\end{array}\right),\left(\begin{array}{ll}
0 & 1 \\
1 & 1
\end{array}\right) \text { and }\left(\begin{array}{ll}
1 & 1 \\
1 & 1
\end{array}\right) .
$$

Only the first code and the last two codes are LCD. It can be seen by hand that the last two LCD codes are equivalent. This means that the first code and the last code are $C\left(\left(Y_{1}, Y_{2}\right)\right)$ and $C\left(\left(Y_{1}^{\prime}, Y_{2}^{\prime}\right)\right)$, respectively, where $Y_{1}=$ $\emptyset, Y_{2}=Y_{1}^{\prime}=Y_{2}^{\prime}=\{1\}$. 
Proposition 3.4. Suppose that $m \geq 1$. Let $C$ be an $L C D[2 m+3,2]$ code with $d\left(C^{\perp}\right) \geq 2$. Then there is a 2 -cover $\left(Y_{1}, Y_{2}\right)$ of an $m$-set $X$ such that $C \cong C^{\prime}\left(\left(Y_{1}, Y_{2}\right)\right)$.

Proof. We may assume without loss of generality that $C$ has generator matrix of the following form:

$$
\left(\begin{array}{lll}
1 & 0 & \\
0 & 1 &
\end{array}\right),
$$

where $M^{\prime}$ is a $2 \times(2 m+1)$ matrix such that no column is $\mathbf{0}_{2}$. If $2 m+1 \geq 4$, then an LCD $[2 m+1,2]$ code is constructed by Lemma 2.3. By continuing this process, an LCD [5,2] code with generator matrix of the form (2) is constructed.

Since no column of $M^{\prime}$ is $\mathbf{0}_{2}$, it is sufficient to consider the $[5,2]$ codes with generator matrices (2), where

$$
\begin{aligned}
M^{\prime}=\left(\begin{array}{lll}
0 & 0 & 0 \\
1 & 1 & 1
\end{array}\right),\left(\begin{array}{lll}
0 & 0 & 1 \\
1 & 1 & 0
\end{array}\right),\left(\begin{array}{lll}
0 & 0 & 1 \\
1 & 1 & 1
\end{array}\right), \\
\\
\left(\begin{array}{lll}
0 & 1 & 1 \\
1 & 0 & 1
\end{array}\right),\left(\begin{array}{lll}
0 & 1 & 1 \\
1 & 1 & 1
\end{array}\right) \text { and }\left(\begin{array}{lll}
1 & 1 & 1 \\
1 & 1 & 1
\end{array}\right) .
\end{aligned}
$$

Only the third code and the last code are LCD. It can be seen by hand that the two LCD codes are equivalent. In addition, the last code is $C^{\prime}\left(\left(Y_{1}, Y_{2}\right)\right)$, where $Y_{1}=Y_{2}=\{1\}$. This completes the proof.

\subsection{LCD codes from 3-covers}

In this subsection, we show that $\operatorname{LCD}[n, 3]$ codes $C$ with $d\left(C^{\perp}\right) \geq 2$ are constructed from 3 -covers for $n=2 m+3,2 m+4(m \geq 1)$.

Proposition 3.5. Suppose that $m \geq 1$. Let $C$ be an $L C D[2 m+3,3]$ code with $d\left(C^{\perp}\right) \geq 2$. Then there is a 3 -cover $\left(Y_{1}, Y_{2}, Y_{3}\right)$ of an m-set $X$ such that $C \cong C\left(\left(Y_{1}, Y_{2}, Y_{3}\right)\right)$.

Proof. We may assume without loss of generality that $C$ has generator matrix of the following form:

$$
\left(\begin{array}{llll}
1 & 0 & 0 & \\
0 & 1 & 0 & M \\
0 & 0 & 1 &
\end{array}\right),
$$


where $M$ is a $3 \times 2 m$ matrix such that no column is $\mathbf{0}_{3}$. If $2 m \geq 8$, then an LCD $[2 m+1,3]$ code is constructed by Lemma 2.3 . By continuing this process, an LCD $[n, 3]$ code with generator matrix of the form (3) is constructed, where $n=5,7,9$. Hence, we show that such a code is constructed from a 3 -cover.

Let $C_{9}$ be an LCD $[9,3]$ code with generator matrix of the form (3) satisfying that all columns of $M$ are distinct. Our computer search shows that $C_{9}$ is equivalent to the code with generator matrix

$$
\left(\begin{array}{lllllllll}
1 & 0 & 0 & 1 & 1 & 1 & 1 & 1 & 1 \\
0 & 1 & 0 & 1 & 0 & 1 & 1 & 0 & 1 \\
0 & 0 & 1 & 1 & 1 & 0 & 1 & 1 & 0
\end{array}\right)
$$

This means that the code is $C\left(\left(Y_{1}, Y_{2}, Y_{3}\right)\right)$, where $Y_{1}=\{1,2,3\}, Y_{2}=\{1,3\}$ and $Y_{3}=\{1,2\}$.

Let $C_{7}$ be an LCD $[7,3]$ code with generator matrix of the form (3) satisfying that all columns of $M$ are distinct. Our computer search shows that $C_{7}$ is equivalent to one of the codes with generator matrices

$$
\left(\begin{array}{lllllll}
1 & 0 & 0 & 1 & 1 & 1 & 1 \\
0 & 1 & 0 & 1 & 1 & 1 & 1 \\
0 & 0 & 1 & 1 & 0 & 1 & 0
\end{array}\right) \text { and }\left(\begin{array}{ccccccc}
1 & 0 & 0 & 1 & 1 & 1 & 1 \\
0 & 1 & 0 & 0 & 1 & 0 & 1 \\
0 & 0 & 1 & 1 & 0 & 1 & 0
\end{array}\right) \text {. }
$$

This means that the codes are $C\left(\left(Y_{1}, Y_{2}, Y_{3}\right)\right)$ and $C\left(\left(Y_{1}^{\prime}, Y_{2}^{\prime}, Y_{3}^{\prime}\right)\right)$, respectively, where $Y_{1}=Y_{2}=Y_{1}^{\prime}=\{1,2\}, Y_{3}=Y_{3}^{\prime}=\{1\}$ and $Y_{2}^{\prime}=\{2\}$.

Our computer search shows that an LCD $[5,3]$ code is equivalent to one of the codes with generator matrices

$$
\left(\begin{array}{lllll}
1 & 0 & 0 & 1 & 1 \\
0 & 1 & 0 & 0 & 0 \\
0 & 0 & 1 & 0 & 0
\end{array}\right),\left(\begin{array}{ccccc}
1 & 0 & 0 & 1 & 1 \\
0 & 1 & 0 & 1 & 1 \\
0 & 0 & 1 & 0 & 0
\end{array}\right) \text { and }\left(\begin{array}{lllll}
1 & 0 & 0 & 1 & 1 \\
0 & 1 & 0 & 1 & 1 \\
0 & 0 & 1 & 1 & 1
\end{array}\right) \text {. }
$$

This means that the codes are $C\left(\left(Y_{1}, Y_{2}, Y_{3}\right)\right), C\left(\left(Y_{1}^{\prime}, Y_{2}^{\prime}, Y_{3}^{\prime}\right)\right)$ and $C\left(\left(Y_{1}^{\prime \prime}, Y_{2}^{\prime \prime}, Y_{3}^{\prime \prime}\right)\right)$, respectively, where $Y_{1}=Y_{1}^{\prime}=Y_{2}^{\prime}=Y_{1}^{\prime \prime}=Y_{2}^{\prime \prime}=Y_{3}^{\prime \prime}=\{1\}$ and $Y_{2}=Y_{3}=$ $Y_{3}^{\prime}=\emptyset$.

Proposition 3.6. Suppose that $m \geq 1$. Let $C$ be an $L C D[2 m+4,3]$ code with $d\left(C^{\perp}\right) \geq 2$. Then there is a 3 -cover $\left(Y_{1}, Y_{2}, Y_{3}\right)$ of an $m$-set $X$ such that $C \cong C^{\prime}\left(\left(Y_{1}, Y_{2}, Y_{3}\right)\right)$. 
Proof. We may assume without loss of generality that $C$ has generator matrix of the following form:

$$
\left(\begin{array}{llll}
1 & 0 & 0 & \\
0 & 1 & 0 & M^{\prime} \\
0 & 0 & 1 &
\end{array}\right)
$$

where $M^{\prime}$ is a $3 \times(2 m+1)$ matrix such that no column is $\mathbf{0}_{3}$. If $2 m+1 \geq 8$, then an LCD $[2 m+2,3]$ code is constructed by Lemma 2.3. By continuing this process, an LCD $[n, 3]$ code with generator matrix of the form (4) is constructed, where $n=6,8,10$.

Let $C_{10}$ be an LCD $[10,3]$ code with generator matrix of the form (44) satisfying that all columns of $M^{\prime}$ are distinct. Our computer search shows that $C_{10}$ is equivalent to the code with generator matrix

$$
\left(\begin{array}{llllllllll}
1 & 0 & 0 & 1 & 1 & 1 & 1 & 1 & 1 & 0 \\
0 & 1 & 0 & 1 & 0 & 1 & 1 & 0 & 1 & 1 \\
0 & 0 & 1 & 1 & 1 & 0 & 1 & 1 & 0 & 1
\end{array}\right)
$$

This means that the code is $C^{\prime}\left(\left(Y_{1}, Y_{2}, Y_{3}\right)\right)$, where $Y_{1}=\{1,2,3\}, Y_{2}=\{1,3\}$ and $Y_{3}=\{1,2\}$.

Let $C_{8}$ be an LCD $[8,3]$ code with generator matrix of the form (41) satisfying that all columns of $M^{\prime}$ are distinct. Our computer search shows that $C_{8}$ is equivalent to the code with generator matrix

$$
\left(\begin{array}{llllllll}
1 & 0 & 0 & 1 & 1 & 1 & 1 & 0 \\
0 & 1 & 0 & 1 & 1 & 1 & 1 & 1 \\
0 & 0 & 1 & 1 & 0 & 1 & 0 & 1
\end{array}\right)
$$

This means that the code is $C^{\prime}\left(\left(Y_{1}, Y_{2}, Y_{3}\right)\right)$, where $Y_{1}=Y_{2}=\{1,2\}$ and $Y_{3}=\{1\}$.

Our computer search shows that an LCD $[6,3]$ code is equivalent to one of the codes with generator matrices $\left(\begin{array}{ll}I_{3} & A\end{array}\right)$, where

$$
A=\left(\begin{array}{lll}
0 & 0 & 0 \\
1 & 1 & 1 \\
1 & 1 & 1
\end{array}\right),\left(\begin{array}{lll}
1 & 1 & 0 \\
0 & 0 & 1 \\
0 & 0 & 1
\end{array}\right) \text { and }\left(\begin{array}{lll}
1 & 1 & 0 \\
1 & 1 & 1 \\
1 & 1 & 1
\end{array}\right) \text {. }
$$

This means that the codes are $C^{\prime}\left(\left(Y_{1}, Y_{2}, Y_{3}\right)\right), C^{\prime}\left(\left(Y_{1}^{\prime}, Y_{2}^{\prime}, Y_{3}^{\prime}\right)\right)$ and $C^{\prime}\left(\left(Y_{1}^{\prime \prime}, Y_{2}^{\prime \prime}, Y_{3}^{\prime \prime}\right)\right)$, respectively, where $Y_{1}=Y_{2}^{\prime}=Y_{3}^{\prime}=\emptyset$ and $Y_{2}=Y_{3}=Y_{1}^{\prime}=Y_{1}^{\prime \prime}=Y_{2}^{\prime \prime}=Y_{3}^{\prime \prime}=$ $\{1\}$. 


\subsection{Remarks}

The elements of an $m$-set $X$ may be taken to be identical. In this case, $X$ is called unlabelled. Let $\mathcal{Y}=\left(Y_{1}, Y_{2}, \ldots, Y_{k}\right)$ be a $k$-cover of $X$. The order of the sets $Y_{1}, Y_{2}, \ldots, Y_{k}$ may not be material. In this case, $\mathcal{Y}$ is called disordered [5].

Proposition 3.7. Let $\mathcal{Y}$ be a $k$-cover of an $m$-set $X$. Let $\mathcal{Y}^{\prime}$ be the $k$-cover obtained from $\mathcal{Y}$ by a permutation of $Y_{1}, Y_{2}, \ldots, Y_{k}$ and a permutation of the elements of $X$. Then $C(\mathcal{Y}) \cong C\left(\mathcal{Y}^{\prime}\right)$.

Proof. Consider the generator matrix $G(\mathcal{Y})$ of $C(\mathcal{Y})$ constructed from a $k$ cover $\mathcal{Y}=\left(Y_{1}, Y_{2}, \ldots, Y_{k}\right)$. A permutation of $Y_{1}, Y_{2}, \ldots, Y_{k}$ implies a permutation of rows of $G(\mathcal{Y})$. A permutation of the elements of $X$ implies a permutation of columns of $G(\mathcal{Y})$. The result follows.

By the above proposition, when we consider codes $C(\mathcal{Y})$ constructed from all $k$-covers $\mathcal{Y}$, which must be checked to achieve a complete classification, it is sufficient to consider only disordered $k$-covers of unlabelled $m$-sets.

Now let us consider LCD codes constructed from 4-covers. Our computer search shows that there are six inequivalent LCD $[6,4]$ codes $D_{6, i}$ $(i=1,2, \ldots, 6)$ with $d\left(D_{6, i}^{\perp}\right) \geq 2$. These codes $D_{6, i}$ have generator matrices $\left(\begin{array}{ll}I_{4} & A\end{array}\right)$, where

$$
\begin{aligned}
A=\left(\begin{array}{ll}
1 & 1 \\
0 & 0 \\
0 & 0 \\
0 & 0
\end{array}\right),\left(\begin{array}{ll}
1 & 1 \\
1 & 1 \\
0 & 0 \\
0 & 0
\end{array}\right), & \left(\begin{array}{ll}
1 & 1 \\
1 & 1 \\
1 & 1 \\
0 & 0
\end{array}\right), \\
& \left(\begin{array}{ll}
1 & 1 \\
1 & 1 \\
1 & 1 \\
1 & 1
\end{array}\right),\left(\begin{array}{ll}
1 & 0 \\
1 & 0 \\
0 & 1 \\
0 & 1
\end{array}\right) \text { and }\left(\begin{array}{ll}
1 & 0 \\
1 & 0 \\
0 & 1 \\
1 & 1
\end{array}\right),
\end{aligned}
$$

respectively. The weight enumerators $W_{6, i}$ of the codes $D_{6, i}$ are listed in Table 1. It is easy to see that the number of disordered 4-covers of an unlabelled 1-set is 4 [5, Table 1]. Only the codes $D_{6, i}(i=1,2,3,4)$ are constructed from 4-covers. 
Table 1: $W_{6, i}(i=1,2, \ldots, 6)$

\begin{tabular}{c|l|c|c}
\hline$i$ & \multicolumn{1}{|c|}{$W_{6, i}$} & $i$ & \multicolumn{1}{|c}{$W_{6, i}$} \\
\hline 1 & $1+3 y+3 y^{2}+2 y^{3}+3 y^{4}+3 y^{5}+y^{6}$ & 4 & $1+6 y^{2}+4 y^{3}+y^{4}+4 y^{5}$ \\
2 & $1+2 y+2 y^{2}+4 y^{3}+5 y^{4}+2 y^{5}$ & 5 & $1+6 y^{2}+9 y^{4}$ \\
3 & $1+y+3 y^{2}+6 y^{3}+3 y^{4}+y^{5}+y^{6}$ & 6 & $1+4 y^{2}+6 y^{3}+3 y^{4}+2 y^{5}$ \\
\hline
\end{tabular}

\section{LCD codes of dimension 2}

It was shown in [7] that

$$
d(n, 2)= \begin{cases}\left\lfloor\frac{2 n}{3}\right\rfloor & \text { if } n \equiv 1,2,3,4 \quad(\bmod 6), \\ \left\lfloor\frac{2 n}{3}\right\rfloor-1 & \text { otherwise }\end{cases}
$$

for $n \geq 2$. Throughout this section, we denote $d(n, 2)$ by $d_{n}$. In this section, we give a classification of LCD $\left[n, 2, d_{n}\right]$ codes for $n=6 t(t \geq 1), 6 t+1$ $(t \geq 1), 6 t+2(t \geq 0), 6 t+3(t \geq 1), 6 t+4(t \geq 0)$ and $6 t+5(t \geq 0)$. In Section 3, we gave some observation of LCD codes of dimension 2, which is established from the concept of 2-covers of $m$-sets. The observation is useful to complete the classification.

Lemma 4.1. Suppose that $n \geq 2$ and $n \equiv 0,1,2,3(\bmod 6)$. If there is an $L C D\left[n, 2, d_{n}\right]$ code $C$, then $d\left(C^{\perp}\right) \geq 2$.

Proof. Write $n=6 t+s$, where $0 \leq s \leq 5$. For $s$ and $d_{n}$, we have the following:

\begin{tabular}{cc|cc|cc}
$s$ & $d_{n}$ & $s$ & $d_{n}$ & $s$ & $d_{n}$ \\
\hline 0 & $4 t-1$ & 2 & $4 t+1$ & 4 & $4 t+2$ \\
1 & $4 t$ & 3 & $4 t+2$ & 5 & $4 t+2$
\end{tabular}

The result follows by Lemma 2.2 ,

Now suppose that $C$ and $C^{\prime}$ are an LCD [2m+2,2] code with $d\left(C^{\perp}\right) \geq 2$ and an LCD $[2 m+3,2]$ code with $d\left(C^{\prime \perp}\right) \geq 2$, respectively, for $m \geq 1$. By Propositions 3.3 and 3.4, we may assume without loss of generality that $C$ 
and $C^{\prime}$ have generator matrices of the following form:

$$
\begin{aligned}
& G^{0}(a, b, c)=\left(\begin{array}{cccc}
1 & 0 & M(a, b, c) & M(a, b, c) \\
0 & 1 &
\end{array}\right) \text { and }
\end{aligned}
$$

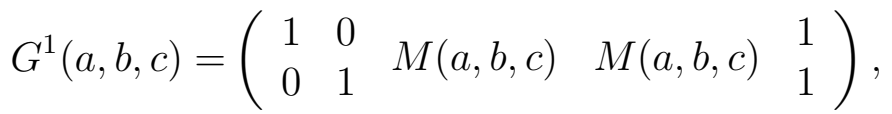

respectively, where

$$
M(a, b, c)=\left(\begin{array}{lll}
\mathbf{1}_{a} & \mathbf{1}_{b} & \mathbf{0}_{c} \\
\mathbf{1}_{a} & \mathbf{0}_{b} & \mathbf{1}_{c}
\end{array}\right)
$$

We denote the codes with generator matrices $G^{0}(a, b, c)$ and $G^{1}(a, b, c)$ by $C^{0}(a, b, c)$ and $C^{1}(a, b, c)$, respectively. Then the codes $C^{\delta}(a, b, c)$ have the following weight enumerators for $\delta \in\{0,1\}$ :

$$
1+y^{1+2(a+b)+\delta}+y^{1+2(a+c)+\delta}+y^{2+2(b+c)} .
$$

For nonnegative integers $a, b, c, n$ and $\delta \in\{0,1\}$, we consider the following conditions:

$$
\begin{aligned}
& d_{n} \leq 1+2(a+b)+\delta \\
& d_{n} \leq 1+2(a+c)+\delta \\
& d_{n} \leq 2+2(b+c) \\
& 2(a+b+c)+2+\delta=n, \\
& b \leq c .
\end{aligned}
$$

We note that the conditions (7)-(9) are related to the minimum weight of $C^{\delta}(a, b, c)$.

Lemma 4.2. (i) Let $S$ be the set of $(a, b, c)$ satisfying the conditions (7)(11), where $\delta=1$.

(1) If $n=6 t+1(t \geq 1)$, then $S=\{(t-1, t, t),(t, t-1, t)\}$.

(2) If $n=6 t+3(t \geq 1)$, then $S=\{(t, t, t)\}$.

(3) If $n=6 t+5(t \geq 1)$, then

$$
S=\left\{\begin{array}{c}
(t-1, t+1, t+1),(t, t, t+1) \\
(t+1, t-1, t+1),(t+1, t, t)
\end{array}\right\}
$$


(ii) Let $S$ be the set of $(a, b, c)$ satisfying the conditions (7)-(11), where $\delta=0$.

(1) If $n=6 t(t \geq 1)$, then $S=\{(t-1, t, t),(t, t-1, t)\}$.

(2) If $n=6 t+2(t \geq 1)$, then $S=\{(t, t, t)\}$.

(3) If $n=6 t+4(t \geq 0)$, then $S=\{(t+1, t, t)\}$.

Proof. All cases are similar, and we only give the details for $n=6 t+1$.

From (9) and (10), we have $a \leq t$. From (7), (8) and (10), we have $t-1 \leq a$. Thus, we have

$$
a \in\{t-1, t\}
$$

Suppose that $a=t-1$. From (7), we have $t \leq b$. From (8), we have $t \leq c$. From (10), we have $b+c=2 t$. Hence, we have $b=c=t$.

Suppose that $a=t$. From (7), we have $t-1 \leq b$. From (8), we have $t-1 \leq c$. From (10), we have $b+c=2 t-1$. From (11), we have $(b, c)=$ $(t-1, t)$.

Lemma 4.3. $C^{\delta}(a, b, c) \cong C^{\delta}(a, c, b)$ for $\delta \in\{0,1\}$.

Proof. The matrix $G^{\delta}(a, c, b)$ is obtained from $G^{\delta}(a, c, b)$ by permutations of rows and columns.

Lemma 4.4. $C^{1}(a, b, c) \cong C^{1}(b, a, c) \cong C^{1}(c, b, a)$.

Proof. We denote the code with generator matrix of the form $M(a, b, c)$ in (5) by $D(a, b, c)$. Let $r_{i}$ be the $i$-th row of $M(a, b, c)$. By considering the matrices $\left(\begin{array}{c}r_{1} \\ r_{1}+r_{2}\end{array}\right)$ and $\left(\begin{array}{c}r_{1}+r_{2} \\ r_{2}\end{array}\right)$, we have $D(a, b, c)=D(b, a, c)=D(c, b, a)$. Since $C^{1}(a, b, c) \cong D(2 a+1,2 b+1,2 c+1)$, the result follows.

The above two lemmas are used for a classification of $\operatorname{LCD}\left[n, 2, d_{n}\right]$ codes.

Theorem 4.5. (i) For $t \geq 1$, there are two inequivalent $L C D[6 t, 2,4 t-1]$ codes.

(ii) For $t \geq 1$, there is a unique $L C D[6 t+1,2,4 t]$ code, up to equivalence.

(iii) For $t \geq 1$, there is a unique $L C D[6 t+2,2,4 t+1]$ code, up to equivalence.

(iv) For $t \geq 1$, there is a unique $L C D[6 t+3,2,4 t+2]$ code, up to equivalence. 
Proof. Let $C$ be an LCD $[n, 2]$ code for $n \geq 4$. For the parameters $[6 t, 2,4 t-$ $1],[6 t+1,2,4 t],[6 t+2,2,4 t+1]$ and $[6 t+3,2,4 t+2](t \geq 1)$, by Lemma 4.1 , we may assume without loss of generality that $C$ has generator matrix of the form $G^{\delta}(a, b, c)$ for $\delta=0,1,0,1$, respectively. In addition, $C$ satisfies (7)(10). By Lemma 4.3, we may assume without loss of generality that $C$ satisfies (11).

(i) Assume that $n=6 t(t \geq 1)$. By Lemma 4.2 (ii), $(a, b, c)$ is $(t-1, t, t)$ or $(t, t-1, t)$. Let $C_{1}$ and $C_{2}$ be the LCD codes with generator matrices $G^{0}(a, b, c)$ for these $(a, b, c)$, respectively. By (6) , the codes $C_{1}$ and $C_{2}$ have the following weight enumerators:

$$
1+2 y^{4 t-1}+y^{4 t+2} \text { and } 1+y^{4 t-1}+y^{4 t}+y^{4 t+1},
$$

respectively. Hence, the two codes are inequivalent.

(ii) Assume that $n=6 t+1(t \geq 1)$. By Lemma 4.2 (i), $(a, b, c)$ is $(t-1, t, t)$ or $(t, t-1, t)$. Let $C_{1}$ and $C_{2}$ be the LCD codes with generator matrices $G^{1}(a, b, c)$ for these $(a, b, c)$, respectively. By Lemma 4.4, $C_{1}$ and $C_{2}$ are equivalent.

(iii) For $n=6 t+2(t \geq 1)$, the uniqueness follows from Lemma 4.2 (ii).

(iv) For $n=6 t+3(t \geq 1)$, the uniqueness follows from Lemma 4.2 (i).

This completes the proof.

We remark that there is a unique $\operatorname{LCD}[3,2,2]$ code, up to equivalence, by Proposition 2.5 .

Lemma 4.6. (i) For $t \geq 0$, there is a unique $L C D[6 t+4,2,4 t+2]$ code $C$ with $d\left(C^{\perp}\right) \geq 2$, up to equivalence.

(ii) For $t \geq 1$, there are two inequivalent $L C D[6 t+5,2,4 t+2]$ codes $C$ with $d\left(C^{\perp}\right) \geq 2$.

Proof. Let $C$ be an LCD $[n, 2]$ code with $d\left(C^{\perp}\right) \geq 2$ and $n \geq 4$. For the parameters $[6 t+4,2,4 t+2](t \geq 0)$ and $[6 t+5,2,4 t+2](t \geq 1)$, since $d\left(C^{\perp}\right) \geq$ 2 , we may assume without loss of generality that $C$ has generator matrix of the form $G^{\delta}(a, b, c)$ for $\delta=0,1$, respectively. In addition, $C$ satisfies (7)-(10). By Lemma 4.3, we may assume without loss of generality that $C$ satisfies (11). 
(i) For $n=6 t+4(t \geq 0)$, the uniqueness follows from Lemma 4.2 (ii).

(ii) Assume that $n=6 t+5(t \geq 1)$. By Lemma 4.2 (i), $(a, b, c)$ is $(t-1, t+$ $1, t+1),(t, t, t+1),(t+1, t-1, t+1)$ or $(t+1, t, t)$. Let $C_{i}(i=1,2,3,4)$ be the LCD codes with generator matrices $G^{1}(a, b, c)$ for these $(a, b, c)$, respectively. By Lemma 4.4, $C_{1} \cong C_{3}$ and $C_{2} \cong C_{4}$. By ([6) , the codes $C_{1}$ and $C_{2}$ have the following weight enumerators:

$$
1+2 y^{4 t+2}+y^{4 t+6} \text { and } 1+y^{4 t+2}+2 y^{4 t+4},
$$

respectively. Hence, the two codes are inequivalent.

This completes the proof.

Remark 4.7. By [7, Theorem 3], the dual codes of the codes given in the above lemma have minimum weight 2 .

Theorem 4.8. (i) For $t \geq 0$, there are two inequivalent $L C D[6 t+4,2,4 t+$ 2] codes.

(ii) For $t \geq 1$, there are four inequivalent $L C D[6 t+5,2,4 t+2]$ codes.

Proof. It is easy to see that all LCD $[n+1, k, d]$ codes $C$ with $d\left(C^{\perp}\right)=1$, which must be checked to achieve a complete classification, can be obtained from all inequivalent LCD $[n, k, d]$ codes.

(i) By Theorem 4.5, there is a unique LCD $[6 t+3,2,4 t+2]$ code, up to equivalence, for $t \geq 1$. The result follows from Lemma 4.6.

(ii) The result follows from Lemma 4.6 and the part (i).

This completes the proof.

We remark that there are three inequivalent LCD $[5,2,2]$ codes (see Table 3]). 


\section{$5 \quad$ LCD codes of dimension 3}

The aim of this section is to establish the following theorem. In Section 3 , we gave some observation of LCD codes of dimension 3, which is established from the concept of 3 -covers of $m$-sets. The observation is useful to do this.

Theorem 5.1. For $n \geq 3$,

$$
d(n, 3)= \begin{cases}\left\lfloor\frac{4 n}{7}\right\rfloor & \text { if } n \equiv 3,5 \quad(\bmod 7) \\ \left\lfloor\frac{4 n}{7}\right\rfloor-1 & \text { otherwise. }\end{cases}
$$

In this section, we also establish the uniqueness of LCD $[n, 3, d(n, 3)]$ codes for $n \equiv 0,2,3,5(\bmod 7)$ and $n \geq 5$.

Throughout this section, we denote $\left\lfloor\frac{4 n}{7}\right\rfloor$ by $\alpha_{n}$.

Lemma 5.2. There is no $L C D\left[n, 3, \alpha_{n}\right]$ code for $n \equiv 2(\bmod 7)$.

Proof. Suppose that there is an (unrestricted) $[n, 3, d]$ code. By the Griesmer bound, we have

$$
n \geq d+\left\lceil\frac{d}{2}\right\rceil+\left\lceil\frac{d}{4}\right\rceil .
$$

Hence, we have

$$
d(n, 3) \leq \begin{cases}\alpha_{n}-1 & \text { if } n \equiv 2 \quad(\bmod 7) \\ \alpha_{n} & \text { otherwise }\end{cases}
$$

The result follows.

Lemma 5.3. Suppose that $n \geq 3$ and $n \equiv 0,4,6(\bmod 7)$. If there is an $L C D\left[n, 3, \alpha_{n}\right]$ code $C$, then $d\left(C^{\perp}\right) \geq 2$.

Proof. Write $n=7 t+s$, where $0 \leq s \leq 6$. For $s$ and $\alpha_{n}$, we have the following:

\begin{tabular}{cc|cc|cc|cc}
$s$ & $\alpha_{n}$ & $s$ & $\alpha_{n}$ & $s$ & $\alpha_{n}$ & $s$ & $\alpha_{n}$ \\
\hline 0 & $4 t$ & 2 & $4 t+1$ & 4 & $4 t+2$ & 6 & $4 t+3$ \\
1 & $4 t$ & 3 & $4 t+1$ & 5 & $4 t+2$ & &
\end{tabular}

The result follows by Lemma 2.2 , 
For nonnegative integers $a, b, c, d, e, f, g, m, \alpha$ and $\delta \in\{0,1\}$, we consider the following conditions:

$$
\begin{aligned}
& \alpha \leq 1+2(a+b+f+g), \\
& \alpha \leq 1+2(a+c+e+g)+\delta, \\
& \alpha \leq 1+2(a+d+e+f)+\delta, \\
& \alpha \leq 2+2(b+c+e+f)+\delta, \\
& \alpha \leq 2+2(b+d+e+g)+\delta, \\
& \alpha \leq 2+2(c+d+f+g), \\
& \alpha \leq 3+2(a+b+c+d), \\
& a+b+c+d+e+f+g=m .
\end{aligned}
$$

Define the following sets:

$$
\begin{aligned}
& R_{1}=\left\{r \in \mathbb{Z} \mid \alpha-m-\frac{3+\delta}{2} \leq r \leq m-\frac{3}{4} \alpha+\frac{3+\delta}{2}\right\}, \\
& R_{2}=\left\{r \in \mathbb{Z} \mid \alpha-m-\frac{4+\delta}{2} \leq r \leq m-\frac{3}{4} \alpha+\frac{2+\delta}{2}\right\} .
\end{aligned}
$$

Lemma 5.4. Let $a, b, c, d, e, f, g$ be nonnegative integers satisfying the conditions (12)-(19).

(i) If $\delta=0$, then $a, e, f, g \in R_{1}$ and $b, c, d \in R_{2}$.

(ii) If $\delta=1$, then $a, f, g \in R_{1}$ and $b, c, d, e \in R_{2}$.

Proof. All cases are similar, and we only give the details for $a \in R_{1}$ and $b \in R_{2}$.

From (15), (16), (17) and (19), we have $a \leq m-\frac{3}{4} \alpha+\frac{3+\delta}{2}$. From (12), (13), (14), (18) and (19), we have $\alpha-m-\frac{3+\delta}{2} \leq a$. Similarly, from (13), (14), (17) and (19), we have $b \leq m-\frac{3}{4} \alpha+\frac{4+\delta}{2}$. From (12), (15), (16), (18) and (19), we have $\alpha-m-\frac{4+\delta}{2} \leq b$. The result follows.

Now suppose that $C$ and $C^{\prime}$ are an LCD $[2 m+3,3]$ code with $d\left(C^{\perp}\right) \geq 2$ and an $\operatorname{LCD}[2 m+4,3]$ code with $d\left(C^{\perp}\right) \geq 2$, respectively for $m \geq 1$. By Propositions 3.5 and 3.6, we may assume without loss of generality that $C$ 
and $C^{\prime}$ have generator matrices of the following form:

$$
\begin{aligned}
& \left(\begin{array}{llllll}
1 & 0 & 0 & & \\
0 & 1 & 0 & M(a, b, c, d, e, f, g) & M(a, b, c, d, e, f, g) \\
0 & 0 & 1 &
\end{array}\right) \text { and } \\
& \left(\begin{array}{llllll}
1 & 0 & 0 & & & 0 \\
0 & 1 & 0 & M(a, b, c, d, e, f, g) & M(a, b, c, d, e, f, g) & 1 \\
0 & 0 & 1 & & 1
\end{array}\right),
\end{aligned}
$$

respectively, where

$$
M(a, b, c, d, e, f, g)=\left(\begin{array}{ccccccc}
\mathbf{1}_{a} & \mathbf{1}_{b} & \mathbf{0}_{c} & \mathbf{0}_{d} & \mathbf{0}_{e} & \mathbf{1}_{f} & \mathbf{1}_{g} \\
\mathbf{1}_{a} & \mathbf{0}_{b} & \mathbf{1}_{c} & \mathbf{0}_{d} & \mathbf{1}_{e} & \mathbf{0}_{f} & \mathbf{1}_{g} \\
\mathbf{1}_{a} & \mathbf{0}_{b} & \mathbf{0}_{c} & \mathbf{1}_{d} & \mathbf{1}_{e} & \mathbf{1}_{f} & \mathbf{0}_{g}
\end{array}\right)
$$

We denote the codes by $C^{0}(a, b, c, d, e, f, g)$ and $C^{1}(a, b, c, d, e, f, g)$, respectively. Then the codes $C^{\delta}(a, b, c, d, e, f, g)$ have the following weight enumerators for $\delta \in\{0,1\}$ :

$$
\begin{aligned}
1 & +y^{1+2(a+b+f+g)}+y^{1+2(a+c+e+g)+\delta}+y^{1+2(a+d+e+f)+\delta} \\
& +y^{2+2(b+c+e+f)+\delta}+y^{2+2(b+d+e+g)+\delta}+y^{2+2(c+d+f+g)}+y^{3+2(a+b+c+d)} .
\end{aligned}
$$

Lemma 5.5. There is no $L C D\left[n, 3, \alpha_{n}\right]$ code for $n \equiv 0,4,6(\bmod 7)$.

Proof. There is no LCD $[4,3,2]$ code (see [7, Table 1]). Assume that $n \equiv$ $0,4,6(\bmod 7)$ and $n \geq 6$. Suppose that there is an LCD $\left[n, 3, \alpha_{n}\right]$ code $C$. By Lemma 5.3, $d\left(C^{\perp}\right) \geq 2$. Hence, $C \cong C^{0}(a, b, c, d, e, f, g)$ if $n \equiv$ $7,11,13(\bmod 14)$ and $C \cong C^{1}(a, b, c, d, e, f, g)$ if $n \equiv 0,4,6(\bmod 14)$ for some $(a, b, c, d, e, f, g)$.

Since $C$ has minimum weight $\alpha_{n},(a, b, c, d, e, f, g)$ satisfies (12)-(19) with $n=3+2 m+\delta$ and $\alpha=\alpha_{n}$.

- $\left(n, \alpha_{n}\right)=(14 t, 8 t)(t \geq 1)$ : We have $R_{2}=\emptyset$, which is a contradiction.

- $\left(n, \alpha_{n}\right)=(14 t+4,8 t+2)(t \geq 1)$ and $(14 t+6,8 t+3)(t \geq 0)$ : We have

$$
(a, b, c, d, e, f, g)=(t, t, t, t, t, t, t)
$$

by Lemma 5.4. These contradict (12) and (19), respectively. 
- $\left(n, \alpha_{n}\right)=(14 t+7,8 t+4)(t \geq 0)$ : We have $R_{1}=\emptyset$, which is a contradiction.

- $\left(n, \alpha_{n}\right)=(14 t+11,8 t+6)$ and $(14 t+13,8 t+7)(t \geq 0)$ : We have

$$
(a, b, c, d, e, f, g)=(t+1, t, t, t, t+1, t+1, t+1)
$$

by Lemma 5.4. These contradict (18) and (19), respectively.

This completes the proof.

Lemma 5.6. There is no $L C D\left[n, 3, \alpha_{n}\right]$ code for $n \equiv 1(\bmod 7)$.

Proof. Assume that $n \equiv 1(\bmod 7)$ and $n \geq 8$. Suppose that there is an LCD $\left[n, 3, \alpha_{n}\right]$ code $C$. Since $n-1 \equiv 0(\bmod 7)$ and $\alpha_{n}=\alpha_{n-1}$, we have

$$
d(n-1,3) \leq \alpha_{n-1}-1=\alpha_{n}-1
$$

by Lemma 5.5. By Lemma 2.2, $d\left(C^{\perp}\right) \geq 2$. Hence, $C \cong C^{0}(a, b, c, d, e, f, g)$ if $n \equiv 1(\bmod 14)$ and $C \cong C^{1}(a, b, c, d, e, f, g)$ if $n \equiv 8(\bmod 14)$ for some $(a, b, c, d, e, f, g)$.

Since $C$ has minimum weight $\alpha_{n},(a, b, c, d, e, f, g)$ satisfies (12)-(19) with $n=3+2 m+\delta$ and $\alpha=\alpha_{n}$.

- $\left(n, \alpha_{n}\right)=(14 t+1,8 t)(t \geq 1)$ : We have

$$
(a, e, f, g)=(t, t, t, t) \text { and } b, c, d \in\{t-1, t\}
$$

by Lemma 5.4. From (19), $b+c+d=3 t-1$. Hence, we have

$$
(b, c, d)=(t-1, t, t),(t, t-1, t) \text { and }(t, t, t-1) .
$$

These contradict (12), (13) and (14), respectively.

- $\left(n, \alpha_{n}\right)=(14 t+8,8 t+4)(t \geq 0):$ We have

$$
a, f, g \in\{t, t+1\} \text { and }(b, c, d, e)=(t, t, t, t)
$$

by Lemma 5.4. From (19), $a+f+g=3 t+2$. Hence, we have

$$
(a, f, g)=(t, t+1, t+1),(t+1, t, t+1) \text { and }(t+1, t+1, t) .
$$

These contradict (18), (15) and (16), respectively. 
This completes the proof.

Hence, from Lemmas 5.2, 5.5 and 5.6, we have

$$
d(n, 3) \leq \alpha_{n}-1,
$$

if $n \equiv 0,1,2,4,6(\bmod 7)$.

Suppose that $C$ is an LCD $\left[n, 3, \alpha_{n}\right]$ code for $n \equiv 3,5(\bmod 7)$ and $n \geq 5$. By Lemmas 2.2, 5.2 and 5.5, $d\left(C^{\perp}\right) \geq 2$. Hence, by Propositions 3.5 and 3.6, $C \cong C^{0}(a, b, c, d, e, f, g)$ if $n \equiv 3,5(\bmod 14)$ and $C \cong C^{1}(a, b, c, d, e, f, g)$ if $n \equiv 10,12(\bmod 14)$ for some $(a, b, c, d, e, f, g)$.

Lemma 5.7. (i) $C^{0}(a, b, c, d, e, f, g) \cong C^{0}(a, b, d, c, e, g, f)$ $\cong C^{0}(a, c, b, d, f, e, g) \cong C^{0}(a, c, d, b, f, g, e) \cong C^{0}(a, d, b, c, g, e, f)$ $\cong C^{0}(a, d, c, b, g, f, e)$.

(ii) $C^{1}(a, b, c, d, e, f, g) \cong C^{1}(a, b, d, c, e, g, f)$.

Proof. The result follows by considering permutations of rows and columns of the generator matrices of $C^{0}(a, b, c, d, e, f, g)$ and $C^{1}(a, b, c, d, e, f, g)$.

By the above lemma, we may assume without loss of generality that

$$
\begin{array}{rll}
b \leq c \leq d & \text { if } & \delta=0, \\
c \leq d & \text { if } & \delta=1 .
\end{array}
$$

Lemma 5.8. Let $S$ be the set of $(a, b, c, d, e, f, g)$ satisfying (12)-(19) and (23).

(i) If $(n, \alpha)=(14 t+3,8 t+1)(t \geq 1)$, then $S=\{(t, t, t, t, t, t, t)\}$.

(ii) If $(n, \alpha)=(14 t+5,8 t+2)(t \geq 0)$, then $S=\{(t+1, t, t, t, t, t, t)\}$.

(iii) If $(n, \alpha)=(14 t+10,8 t+5)(t \geq 0)$, then $S=\{(t+1, t, t, t, t, t+1, t+1)\}$.

(iv) If $(n, \alpha)=(14 t+12,8 t+6)(t \geq 0)$, then

$$
S=\{(t+1, t+1, t, t, t, t+1, t+1)\} .
$$

Proof. All cases are similar, and we only give the details for (iv), which is the complicated case. 
Suppose that $(n, \alpha)=(14 t+12,8 t+6)(t \geq 0)$. By Lemma 5.4, $R_{1}=$ $R_{2}=\{t, t+1\}$. From (12), $4 t+\frac{5}{2} \leq a+b+f+g$. Hence, we have

$$
|\{s \in\{a, b, f, g\} \mid s=t+1\}| \geq 3 .
$$

From (19), $a+b+c+d+e+f+g=7 t+4$. Hence, we have

$$
|\{s \in\{a, b, c, d, e, f, g\} \mid s=t+1\}|=4 .
$$

Therefore, we have

$$
(a, b, f, g) \in\left\{\begin{array}{l}
(t+1, t+1, t+1, t),(t+1, t+1, t, t+1), \\
(t+1, t, t+1, t+1),(t, t+1, t+1, t+1), \\
(t+1, t+1, t+1, t+1)
\end{array}\right\} .
$$

Here, we remark that

$$
|\{s \in\{c, d, e\} \mid s=t+1\}| \leq 1 .
$$

- $(a, b, f, g)=(t+1, t+1, t+1, t)$ : From (13), (16) and (17), we have

$$
2 t+1 \leq c+e, 2 t+\frac{1}{2} \leq d+e \text { and } 2 t+1 \leq c+d,
$$

respectively. This contradicts (24).

- $(a, b, f, g)=(t+1, t+1, t, t+1)$ : From (14), (15) and (17), we have

$$
2 t+1 \leq d+e, 2 t+\frac{1}{2} \leq c+e \text { and } 2 t+1 \leq c+d,
$$

respectively. This contradicts (24).

- $(a, b, f, g)=(t+1, t, t+1, t+1)$ : From (15), (16) and (18), we have

$$
2 t+\frac{1}{2} \leq c+e, 2 t+\frac{1}{2} \leq d+e \text { and } 2 t+\frac{1}{2} \leq c+d,
$$

respectively. This contradicts (24).

- $(a, b, f, g)=(t, t+1, t+1, t+1)$ : From (13), (14) and (18), we have

$$
2 t+1 \leq c+e, 2 t+1 \leq d+e \text { and } 2 t+\frac{1}{2} \leq c+d,
$$

respectively. This contradicts (24). 
The result follows.

Therefore, we have the following theorem.

Theorem 5.9. For $n \equiv 3,5(\bmod 7)$ and $n \geq 5$, there is a unique $L C D$ $\left[n, 3, \alpha_{n}\right]$ code, up to equivalence.

From (22), we have $d(n, 3) \leq \alpha_{n}-1$ if $n \equiv 0,1,2,4,6(\bmod 7)$. Now we construct an LCD code meeting the bound. Suppose that $C$ is an LCD $\left[n, 3, \alpha_{n}-1\right]$ code for $n \equiv 0,2(\bmod 7)$ and $n \geq 7$. By Lemmas 2.2 , 5.2 and 5.5. $d\left(C^{\perp}\right) \geq 2$. Hence, by Propositions 3.5 and 3.6. $C \cong C^{0}(a, b, c, d, e, f, g)$ if $n \equiv 7,9(\bmod 14)$ and $C \cong C^{1}(a, b, c, d, e, f, g)$ if $n \equiv 0,2(\bmod 14)$ for some $(a, b, c, d, e, f, g)$.

Lemma 5.10. Let $S$ be the set of $(a, b, c, d, e, f, g)$ satisfying (12)-(19) and (23) .

(i) If $(n, \alpha)=(14 t, 8 t-1)(t \geq 1)$, then

$$
S=\{(t, t-1, t, t, t-1, t, t),(t, t-1, t-1, t, t, t, t)\}
$$

(ii) If $(n, \alpha)=(14 t+2,8 t)(t \geq 1)$, then

$$
S=\{(t, t, t, t, t-1, t, t),(t, t, t-1, t, t, t, t)\} .
$$

(iii) If $(n, \alpha)=(14 t+7,8 t+3)(t \geq 0)$, then

$$
S=\left\{\begin{array}{l}
(t, t, t, t, t+1, t, t+1),(t, t, t, t, t+1, t+1, t), \\
(t, t, t, t, t, t+1, t+1)
\end{array}\right\} .
$$

(iv) If $(n, \alpha)=(14 t+9,8 t+4)(t \geq 0)$, then

$$
S=\left\{\begin{array}{l}
(t+1, t, t, t, t+1, t, t+1),(t+1, t, t, t, t+1, t+1, t), \\
(t+1, t, t, t, t, t+1, t+1)
\end{array}\right\} .
$$

Proof. All cases are similar, and we only give the details for (i).

Suppose that $(n, \alpha)=(14 t, 8 t-1)(t \geq 1)$. By Lemma 5.4, $R_{1}=R_{2}=$ $\{t-1, t\}$. From (19),$a+b+c+d+e+f+g=7 t-2$. Hence, we have

$$
|\{s \in\{a, b, c, d, e, f, g\} \mid s=t-1\}|=2 .
$$


From (12), (13) and (14), we have

$$
\begin{aligned}
& 4 t-1 \leq a+b+f+g, \\
& 4 t-\frac{3}{2} \leq a+c+e+g \text { and } \\
& 4 t-1 \leq a+d+e+f
\end{aligned}
$$

respectively.

Now suppose that $a=t-1$. From (26), we have $b=c=d=e=f=$ $g=t$. Since this contradicts (25), we have $a=t$. Suppose that $g=t-1$. From (26), we have $b=c=d=e=f=t$. Since this contradicts (25), we have $g=t$. From (17), we have

$$
4 t-\frac{3}{2} \leq c+d+f+g
$$

Suppose that $f=t-1$. From (26) and (27), we have $b=c=d=e=t$. Since this contradicts (25), we have $f=t$. Suppose that $d=t-1$. From (27), we have $c=t$, which contradicts (23). Therefore, we have

$$
(b, c, e) \in\{(t-1, t-1, t),(t-1, t, t-1)\} .
$$

The result follows.

We denote the code with generator matrix of the form $M(a, b, c, d, e, f, g)$ in (20) by $D(a, b, c, d, e, f, g)$. It is trivial that $C^{0}(a, b, c, d, e, f, g) \cong D(2 a, 2 b+$ $1,2 c+1,2 d+1,2 e, 2 f, 2 g)$ and $C^{1}(a, b, c, d, e, f, g) \cong D(2 a, 2 b+1,2 c+1,2 d+$ $1,2 e+1,2 f, 2 g)$.

Lemma 5.11. (i) For $t \geq 1, D(2 t, 2 t-1,2 t+1,2 t+1,2 t-1,2 t, 2 t) \cong$ $D(2 t, 2 t-1,2 t-1,2 t+1,2 t+1,2 t, 2 t)$.

(ii) For $t \geq 1, D(2 t, 2 t+1,2 t-1,2 t+1,2 t+1,2 t, 2 t) \cong D(2 t, 2 t+1,2 t+$ $1,2 t+1,2 t-1,2 t, 2 t)$.

Proof. Let $r_{i}$ be the $i$-th row of $M(a, b, c, d, e, f, g)$. Consider the following matrices:

$$
\left(\begin{array}{c}
r_{1} \\
r_{3} \\
r_{2}+r_{3}
\end{array}\right) \text { and }\left(\begin{array}{c}
r_{1} \\
r_{2} \\
r_{2}+r_{3}
\end{array}\right)
$$

for (i) and (ii), respectively. The result follows. 
Theorem 5.12. For $n \equiv 0,2(\bmod 7)$ and $n \geq 7$, there is a unique $L C D$ $\left[n, 3, \alpha_{n}-1\right]$ code, up to equivalence.

Proof. The result follows from Lemmas [5.7, 5.10 and 5.11.

Lemma 5.13. There is an $\operatorname{LCD}\left[n, 3, \alpha_{n}-1\right]$ code for $n \equiv 1,4,6(\bmod 7)$ and $n \geq 4$.

Proof. There is an LCD $[4,3,2]$ code (see [7, Table 1]). Suppose that $n \geq 6$. Consider the following codes:

$$
\begin{aligned}
& C^{1}(t+1, t, t, t, t, t, t), \\
& C^{1}(t, t, t, t, t, t+1, t+1), \\
& C^{0}(t+1, t, t, t, t+1, t+1, t+1), \\
& C^{0}(t+1, t+1, t, t, t+1, t+1, t+1), \\
& C^{0}(t+1, t+1, t, t+1, t+1, t+1, t+1) \text { and } \\
& C^{1}(t+1, t+1, t+1, t+1, t+1, t+1, t+1),
\end{aligned}
$$

for $t \geq 0$. We denote these codes by $C_{i}(i=1,2, \ldots, 6)$, respectively. The codes $C_{i}$ have lengths $14 t+6,14 t+8,14 t+11,14 t+13,14 t+15$ and $14 t+18$, respectively. The weight enumerators $W_{i}$ of $C_{i}(i=1,2, \ldots, 6)$ are obtained by (21), where $W_{i}$ are listed in Table 2, The result follows.

Remark 5.14. For the parameters $[4,3,1],[6,3,2]$ and $[8,3,3]$, a number of inequivalent LCD codes are known (see Table 3).

Table $2: W_{i}(i=1,2, \ldots, 6)$

\begin{tabular}{c|c|c|c}
\hline$i$ & $W_{i}$ & $i$ & $W_{i}$ \\
\hline 1 & $1+y^{8 t+2}+3 y^{8 t+3}+2 y^{8 t+4}+y^{8 t+5}$ & 4 & $1+y^{8 t+6}+3 y^{8 t+7}+2 y^{8 t+8}+y^{8 t+9}$ \\
2 & $1+y^{8 t+3}+2 y^{8 t+4}+3 y^{8 t+5}+y^{8 t+6}$ & 5 & $1+y^{8 t+7}+2 y^{8 t+8}+3 y^{8 t+9}+y^{8 t+10}$ \\
3 & $1+y^{8 t+5}+3 y^{8 t+6}+3 y^{8 t+7}$ & 6 & $1+y^{8 t+9}+3 y^{8 t+10}+3 y^{8 t+11}$ \\
\hline
\end{tabular}

Lemmas 5.2, 5.5, 5.6, 5.13 and Theorems 5.9, 5.12 complete the proof of Theorem 5.1 . 


\section{Classification of LCD codes for small pa- rameters}

In this section, we give a complete classification of LCD $[n, k]$ codes having the minimum weight $d(n, k)$ for $2 \leq k \leq n-1 \leq 15$.

We describe how LCD $[n, k]$ codes having the minimum weight $d(n, k)$ were classified. Let $d_{\text {all }}(n, k)$ denote the largest minimum weight among all (unrestricted) $[n, k]$ codes. The values $d_{\text {all }}(n, k)$ can be found in [8]. For a fixed pair $(n, k)$, we found all inequivalent $[n, k]$ codes by one of the following methods. If there is no LCD $\left[n, k, d_{\text {all }}(n, k)\right]$ code, then we consider the case $d_{\text {all }}(n, k)-1$.

Let $C$ be an $[n, k, d]$ code with parity-check matrix $H$. Let $D$ be a code with parity-check matrix obtained from $H$ by deleting a column. The code $D$ is an $\left[n-1, k-1, d^{\prime}\right]$ code with $d^{\prime} \geq d$. By considering the inverse operation, all $[n, k, d]$ codes are obtained from $\left[n-1, k-1, d^{\prime}\right]$ codes with $d^{\prime} \geq d$. Starting from $\left[n, 1, d^{\prime}\right]$ codes with $d^{\prime} \geq d$, all $[n+t, 1+t, d]$ codes are found for a given $t \geq 1$. This was done by adding one column at a time, and complete equivalence tests are carried out for each new column added. It is obvious that all codes, which must be checked to achieve a complete classification, can be obtained.

For some parameters, we employ the following method, due to the computational complexity. Every $[n, k, d]$ code is equivalent to a code with generator matrix of the form $\left(\begin{array}{ll}I_{k} & A\end{array}\right)$, where $A$ is a $k \times(n-k)$ matrix. The set of matrices $A$ was constructed, row by row. Permuting the rows and columns of $A$ gives rise to different generator matrices which generate equivalent codes. Here, we consider a natural (lexicographical) order $<$ on the set of the vectors of length $n-k$. Let $r_{i}$ be the $i$-th row of $A$. We consider only matrices $A$, satisfying the condition $r_{1}<r_{2}<\cdots<r_{k}$ and $\operatorname{wt}\left(r_{i}\right) \geq d-1$ if $d \geq 3$ and the condition $r_{1} \leq r_{2} \leq \cdots \leq r_{k}$ and $\operatorname{wt}\left(r_{i}\right) \geq d-1$ if $d \leq 2$. It is obvious that all codes, which must be checked to achieve a complete classification, can be obtained.

For $2 \leq k \leq n-1 \leq 15$, the numbers $N(n, k, d(n, k))$ of the inequivalent LCD $[n, k, d(n, k)]$ codes are listed in Table 3, along with the values $d(n, k)$. All generator matrices of the codes in the table can be obtained electronically from http://www .math.is.tohoku .ac.jp/ mharada/LCD/.

We continue a classification of LCD codes with parameters $[2 m+3,2 m, 2]$ and $[2 m+4,2 m+1,2]$. In Proposition 3.5, for an LCD $[2 m+3,2 m, 2]$ code $C$, 
Table 3: $(d(n, k), N(n, k, d(n, k)))$

\begin{tabular}{c|ccccccc}
\hline$n \backslash k$ & 2 & 3 & 4 & 5 & 6 & 7 & 8 \\
\hline 3 & $(2,1)$ & & & & & & \\
4 & $(2,2)$ & $(1,2)$ & & & & & \\
5 & $(2,3)$ & $(2,1)$ & $(2,1)$ & & & & \\
6 & $(3,2)$ & $(2,3)$ & $(2,4)$ & $(1,3)$ & & & \\
7 & $(4,1)$ & $(3,1)$ & $(2,9)$ & $(2,2)$ & $(2,1)$ & & \\
8 & $(5,1)$ & $(3,3)$ & $(3,1)$ & $(2,9)$ & $(2,6)$ & $(1,4)$ & \\
9 & $(6,1)$ & $(4,1)$ & $(4,1)$ & $(3,2)$ & $(2,23)$ & $(2,3)$ & $(2,1)$ \\
10 & $(6,2)$ & $(5,1)$ & $(4,5)$ & $(3,11)$ & $(3,2)$ & $(2,23)$ & $(2,9)$ \\
11 & $(6,4)$ & $(5,6)$ & $(4,20)$ & $(4,4)$ & $(4,1)$ & $(3,1)$ & $(2,51)$ \\
12 & $(7,2)$ & $(6,1)$ & $(5,6)$ & $(4,37)$ & $(4,11)$ & $(3,22)$ & $(2,396)$ \\
13 & $(8,1)$ & $(6,6)$ & $(6,2)$ & $(5,5)$ & $(4,146)$ & $(4,4)$ & $(3,27)$ \\
14 & $(9,1)$ & $(7,1)$ & $(6,16)$ & $(5,101)$ & $(5,4)$ & $(4,301)$ & $(4,8)$ \\
15 & $(10,1)$ & $(7,8)$ & $(6,89)$ & $(6,10)$ & $(6,2)$ & $(5,1)$ & $(4,985)$ \\
16 & $(10,2)$ & $(8,1)$ & $(7,7)$ & $(6,283)$ & $(6,60)$ & $(5,1596)$ & $(5,1)$ \\
\hline$n \backslash k$ & 9 & 10 & 11 & 12 & 13 & 14 & 15 \\
\hline 10 & $(1,5)$ & & & & & & \\
11 & $(2,4)$ & $(2,1)$ & & & & & \\
12 & $(2,51)$ & $(2,12)$ & $(1,6)$ & & & & \\
13 & $(2,619)$ & $(2,103)$ & $(2,5)$ & $(2,1)$ & & & \\
14 & $(3,31)$ & $(2,1370)$ & $(2,103)$ & $(2,16)$ & $(1,7)$ & & \\
15 & $(4,2)$ & $(3,34)$ & $(2,2143)$ & $(2,196)$ & $(2,7)$ & $(2,1)$ & \\
16 & $(4,1772)$ & $(4,7)$ & $(3,34)$ & $(2,4389)$ & $(2,196)$ & $(2,20)$ & $(1,8)$ \\
\hline
\end{tabular}

there is a 3 -cover $\left(Y_{1}, Y_{2}, Y_{3}\right)$ such that $C^{\perp} \cong C\left(\left(Y_{1}, Y_{2}, Y_{3}\right)\right)$. In addition, by Proposition 3.7, when we consider codes $C(\mathcal{Y})$ constructed from all $k$-covers $\mathcal{Y}$, which must be checked to achieve a complete classification, it is sufficient to consider only disordered $k$-covers of unlabelled $m$-sets. According to [5], let $\operatorname{Tdu}(m, k)$ denote the number of disordered $k$-covers of an unlabelled $m$ set. The formula $\operatorname{Tdu}(m, k)$ is given in [5, Theorem 2]. For $m \leq 7$ and $k \leq 8, \operatorname{Tdu}(m, k)$ is numerically determined in [5, Table 1] (see also A005783 in [14]). Our computer search shows the following:

Proposition 6.1. If $1 \leq m \leq 11$, then

$$
N(2 m+3,2 m, 2)=N(2 m+4,2 m+1,2)=\mathrm{Tdu}(m, 3) .
$$

Acknowledgment. This work was supported by JSPS KAKENHI Grant Number 15H03633. The authors would like to thank Makoto Araya for his 
useful discussions. The authors would also like to thank Yuta Watanabe and the anonymous referees for helpful comments.

\section{References}

[1] W. Bosma, J. Cannon and C. Playoust, The Magma algebra system I: The user language, J. Symbolic Comput. 24 (1997), 235-265.

[2] C. Carlet and S. Guilley, Complementary dual codes for countermeasures to side-channel attacks, In: E.R. Pinto et al. (eds.), Coding Theory and Applications, CIM Series in Mathematical Sciences, vol. 3, pp. 97-105, Springer, 2014.

[3] C. Carlet, S. Mesnager, C. Tang and Y. Qi, Euclidean and Hermitian LCD MDS codes, Des. Codes Cryptogr., (to appear), https://doi.org/10.1007/s10623-018-0463-8, arXiv:1702.08033.

[4] C. Carlet, S. Mesnager, C. Tang, Y. Qi and R. Pellikaan, Linear codes over $\mathbb{F}_{q}$ are equivalent to LCD codes for $q>3$, IEEE Trans. Inform. Theory 64 (2018), 3010-3017.

[5] R.J. Clarke, Covering a set by subsets, Discrete Math. 81 (1990), 147152.

[6] S.T. Dougherty, J.-L. Kim, B. Ozkaya, L. Sok and P. Solé, The combinatorics of LCD codes: linear programming bound and orthogonal matrices, Int. J. Inf. Coding Theory 4 (2017), 116-128.

[7] L. Galvez, J.-L. Kim, N. Lee, Y.G. Roe and B.-S. Won, Some bounds on binary LCD codes, Cryptogr. Commun. 10 (2018), 719-728.

[8] M. Grassl, Code tables: Bounds on the parameters of various types of codes, Available online at http://www.codetables.de/, Accessed on 2018-01-22.

[9] C. G̈uneri, B. Özkaya and P. Solé, Quasi-cyclic complementary dual codes, Finite Fields Appl. 42 (2016), 67-80.

[10] L. Jin, Construction of MDS codes with complementary duals, IEEE Trans. Inform. Theory 63 (2017), 2843-2847. 
[11] J.L. Massey, Linear codes with complementary duals, Discrete Math. 106/107 (1992), 337-342.

[12] S. Mesnager, C. Tang and Y. Qi, Complementary dual algebraic geometry codes, IEEE Trans. Inform. Theory 64 (2018), 2390-2397.

[13] M. Sendrier, Linear codes with complementary duals meet the GilbertVarshamov bound, Discrete Math. 285 (2004), 345-347.

[14] N. Sloane and S. Plouffe, The Encyclopedia of Integer Sequences, Academic Press, San Diego, CA, 1995 (Available online at https://oeis.org). 